\title{
Knowledge-Based Recognition of Crosshatched Areas in Engineering Drawings
}

\author{
S.Ablameyko, V.Bereishik, O.Frantskevich, M.Homenko, N.Paramonova \\ Institute of Engineering Cybernetics, Belarussian Academy of Sciences \\ 6 Surganova Str, 220012, Minsk, Republic of Belarus \\ Telephone: 375 (172) $68-51-44$ \\ Fax: 375 (172) 31-84-03 \\ Email: abl@newman.basnet.minsk.by
}

\begin{abstract}
One of the main tasks in the problem of engineering drawing (ED) automatic input and interpretation is recognition of ED primitives to obtain $2 \mathrm{D}$ image representation for further use in CAD systems. This paper presents two-stage knowledge-based algorithm to recognize crosshatched areas in ED images. At the first stage, pure segment chains i.e. simple structure crosshatched area parts satisfying a template description are detected. At the second stage, the final assembly of the area takes place by using the chains assembled at the first stage.
\end{abstract}

\section{Introduction}

The problem of fast, precise and inexpensive input of paper-based engineering drawings (ED) into Computer-Aided Design (CAD) systems is very important for many industrial applications. During last decade scanners started to be widely used for this task that allows one to fast transform drawings into digital raster form but usually requires a highly developed software to convert scanned images into high-level models. The desirable output of this transformation for engineering drawings would be a 3D model of an object drawn at ED projections. There exist papers discussed such a transformation although there are not known automatic solutions of this task. As an intermediate step could be an automatic transformation of EDs into models containing drawing representation in terms of graphic primitives or entities suitable for CAD systems.

There are many systems that perform automatic image vectorization and allow one to represent images in terms of simple graphic primitives although there are very few systems with attempts to recognize automatically engineering drawing primitives and entities. The systems described in [1-3] are successfully used for processing and vectorization of ED images. ANON and CELLESTIN systems have been developed for recognition of several primitives from mechanical drawings $[4,7]$.

One of the more important tasks in interpretation of engineering drawings is recognition of main ED entities: hatched lines, circular arcs, blocks, crosshatched areas, dimensions and others. This is a difficult task and there are only research papers (not commercial systems) devoted to its solution.

The approaches to recognize crosshatched areas were developed in French systems CIPLAN, REDRAW, CELLESTIN [4,9,10]. A priori knowledge is intensively used for this task. At first, parallel lines having the same slope angle and 
thickness are extracted on a raster image. They form a low level of an image model. Then, the image is vectorized and crosshatched areas are collected by using information from the low level. Approaches used in these systems are different by mainly of high level specifics.

In paper [11], crosshatched areas on a land register map are extracted on a raster image. During building and image graph, a closed polygon is searched. If the search fails, an operator is asked for a help. Finally, all nodes and edges internal to the perimeter, which are recognized as parts of hatching lines, are removed from the image graph. A self-supervised approach that includes a spacing estimation step and a recognition step to extract section hatching lines is described in paper [8].

In this paper, we propose a fast algorithm for recognition of crosshatched areas in engineering drawings that has been developed in frame of our system for ED interpretation [5,6]. At first, we show specifics of ED image vectorization (in difference from direct vectorization technology) that allows to take into account all specifics of ED images. Then, the two-stage recognition algorithm is proposed that is based on maximal use of knowledge. At the first stage, hypotheses are formulated as to whether the segments belong to an area boundary or an area internal line using a local analysis of relations between the segments. At the second stage, the final assembly of the area takes place, taking into account the chains assembled at the first stage.

\section{ED Image Vectorization}

Usually, the technology of ED entities recognition includes two basic processes: image vectorization and ED entities recognition based on the vectorized image. Description of algorithms used for binary image vectorization is given in our paper [6] that have been firstly developed and successfully used for vectorization of map images. However, every image type has its own specifics and let us show how they could be applied to vectorize ED images. The vectorization of ED images includes the following stages.

1. At first, engineering drawing is scanned and transformed into binary raster format. Binarization could be made in a scanner or by software if a quality of the original image is not high. The image file format could be a bit-map or compressed one. We use a modified run-length binary image representation [6].

2. Noise reduction is performed.

3. Thickness of image lines is evaluated by histogram building. Such as a set of ED lines can be mainly divided into thin and thick ones, two picks are extracted on the histogram that correspond to these two line classes. Using this information, maximal thickness of thin lines and minimal thickness of thick lines is calculated.

4. Contours of image connected components are extracted and their parameters (bounding rectangle, area, perimeter) are computed. Potential text symbols are extracted based on the computed parameters.

5. Assembly of detected text symbols into text strings is performed based on the distance between text symbols.

6. Using the defined on the stage 3 line thickness value, a morphological operation "erosion" with a structuring element in form of a circle whose radius is equal to a half of the maximal thin line thickness plus one is performed. It allows to delete the thin lines from the image and transform arrowheads into isolated objects 
that can be easily further recognized by using their shape. In a result, potential arrowheads are extracted.

7. If ED image contains large black areas, thick lines are deleted by using the same technique as on the previous stage. Then, a mathematical operation "dilation" is used to reconstruct remained connected components that correspond to the large black areas.

8. Thinning of the image obtained in the second stage is performed. During this operation line thickness in every pixel is also computed.

9.Feature points (nodes and end points) and segments bounded by feature points are extracted from the thinned image and vectorized. Under segment extraction, its primary approximation using Freeman code is performed. Simultaneously some segment characteristics are computed including an average segment thickness and structural noise (defects) is removed using segment length and type.

10.Segment approximation by straight lines and circular arcs is performed. The information about segment form is used as hypothesis for geometrical primitive extraction.

In a result, skeleton (S-form) and contour (C-form) forms of vector image representation are obtained and used further for object recognition.

\section{The Recognition Algorithm \\ 3.1.Main Principles and Stages}

For interpretation of ED images we developed main principles that can be considered as different sides of the interpretation process, defining a technology and scheme of main algorithms. They are: "from simple to complex", "from local to global analysis", "hypotheses for ED entities recognition", "engineering drawing specifics". These general principles are used in the proposed algorithm although the following particular principles for crosshatched area recognition have been proposed:

- a priori knowledge about an engineering drawing is used;

- groups of parallel lines are detected;

- closed areas are formed in a vector image and sought-for areas are recognized through an analysis of area attributes;

- area extraction is performed in a vector image.

In our algorithm, a crosshatched area is recognized in two stages. At the first stage, pure segment chains, i.e. crosshatched area parts satisfying a template description are detected. For this aim, hypotheses are formulated as to whether the Sform segments belong to an area boundary or an area internal line using a local analysis of relations between the segments. The hypotheses are tested as an attempt is made to assembly the segments into pure chains. At the second stage, the final assembly of areas takes place taking into account the pure chains assembled at the first stage. For this purpose, the search region is expanded and the segments are subjected to a more detailed analysis by using the relations between them.

\subsection{Search of Area and Extraction of Pure Chains}

In the beginning, search of crosshatched area in a drawing is performed by using segment analysis and their relations. The hypothesis about the potential area beginning is formed if there exist two segments (L) that satisfy the template 
description of a line in a hatched area, that are interconnected by a third segment or by a group of segments (B), and that are associated by what we call template (E) relations. The template description of a line includes its width, the angle it forms with the coordinate axes, and the type of the segment of which the line is a part. The template relations are as follows:

- the segments $\mathrm{L}$ are parallel,

- each segment $L$ is adjacent to a boundary segment $\mathbf{B}$,

- the segments Ls are located on one side of a segment $\mathbf{B}$,

- it is possible to drop a perpendicular from one segment $\mathbf{L}$ to another,

- the spacing between the segments $\mathbf{L}$ is within the allowable limits.

A combination of the type $\mathbf{E}(\mathbf{L B L})$ is tentatively regarded as a candidate for the beginning of area assembly (Fig. 1).

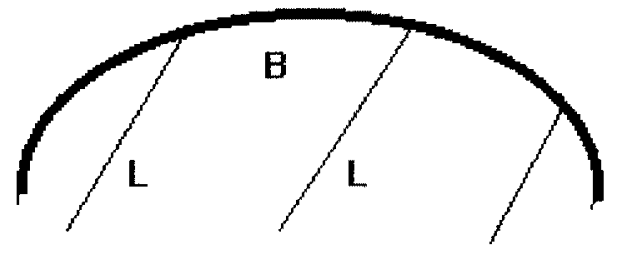

Fig.1. Beginning of crosshatching area recognition.

To test the hypothesis, we attempt to extend the combination in both directions, that is, to assembly pure chains. For this purpose, we choose the pair LB or BL, determine the tracing direction, and test all the segments related to the starting combination in the specified area. If pairs of the type BL are encountered, we recognize as best the pair that meets the following requirements: it points in the same direction as the area that is traced, the segments remain constant in width, the segments $\mathbf{B}$ form the smallest angle at the junctions in the specified direction, and the deviation in spacing between the segments $L s$ is minimal and stays within the specified limits.

A pure chain is considered assembled if the starting combination is extended at least to the form $\mathbf{E}(\mathbf{L B L B L})$. The assembly of a pure chain is discontinued if:

- a change has occurred in the width of the segments B,

- the chain has moved outside the specified search area,

- a complex branching has taken place,

- the path has broken off,

- the chain has moved onto an already assembled part of the area.

Every assembled chain has a particular average spacing between the lines $\mathbf{L}$ and a particular angle of $\mathbf{L}$ incline. Moreover, it preserves its connection to the original vectur model (the $\mathbf{S}$-form) and particular relations to segments are defined.

\subsection{Extraction of Full Crosshatched Areas}

Input information for the second stage is pure chains assembled at the previous stage and remained segments. As part of the search for the extension of a combination 
of the BL type, an extension search tree is constructed, starting from the first (last) segment of the chain. It includes all segments associated with the initial segments $\mathbf{B}$ and $\mathbf{L}$ and falling within the seal $\mathrm{h}$ ar $\mathbf{a}$. All segments of arbitrary width and running parallel to the original segment $\mathbf{L}$ are tried as candidates for the role of $\mathbf{L}$, and all segments interconnecting the candidates to $\mathrm{L}$ are tried as candidates for the role of $\mathbf{B}$. Importantly, one and the same segment can be treated as a candidate for the role of both $\mathbf{L}$ and $\mathbf{B}$. In contrast to the first stage, the requirements to be met by the candidates are not as stringent because the vector model may be somewhat distorted as compared to the original image. As the tree is constructed, every incoming segment is characterized in terms of several parameters. The key parameters are the similitude to $\mathbf{L}$, the deviation (if any) from the original tracing direction, width, and the angle of deviation from the ancestor in the tree. For segments similar to $\mathbf{L}$, these parameters are: the type of relation with the ancestor in the path tree (intersection, adjacency), affiliation with the same area as the preceding segment $\mathbf{L}$, and the area characteristics if a segment belongs to a pure chain or to an already assembled hatched area. As the path tree is constructed, candidates for the role of a line in the crosshatching area are identified.

Every tree level, that is, every likely path, is analyzed to see if it can be extendud further; it is terminated if it continues beyond the search area or if an assembled part of a hatched area is detected. After all the possible paths are terminated, combinations of the type $\mathbf{B L}$ are analyzed, and a characterization is formed for the entire path. From comparative analysis of the combinations of characteristics of all paths, it is possible to choose the most probable from the viewpoint of drawing rules. As a result, any one of the following alternative decisions can be chosen:

- to continue the chain,

- to merge chains,

- to effect closure.

With the first alternative, the chain is assembled in the usual manner. With the second alternative, chains similar in parameters, that is, constituents of the same area, are combined. With the third alternative, the assembly of the area boundary is completed so that the segments Ls are assembled into the area lines with the aid of graphic primitives. If a hatched area has internal hollows, the external and internal boundaries of the area are combined into a single block.

\section{Experimental Results}

The software for ED interpretation that includes the algorithm for crosshatched area recognition has been developed on IBM PC computer in C language. The input binary images are obtained from engineering drawings with size A4-A2. The raster data for the processing are represented in PCX or TIFF formats. Output data are usually represented in DXF files although other formats could be used too.

The vectorization and recognition process has been tested on many ED images. Example of an initial image is shown in Fig. 2 and its vectorized form is shown in Fig.3. The result of crosshatching area recognition of the drawing is shown in Fig.4. After the entities recognition, they are aligned and transformed to DXF AutoCAD format. The performed experiments show enough good quality and noise stability of recognition. The vectorization time for the image shown in Fig. 1 is equal to $30 \mathrm{sec}$ on 
IBM PC Pentium. Time for recognition of crosshatched areas in this drawing from the vectorized model is equal to $30 \mathrm{sec}$.

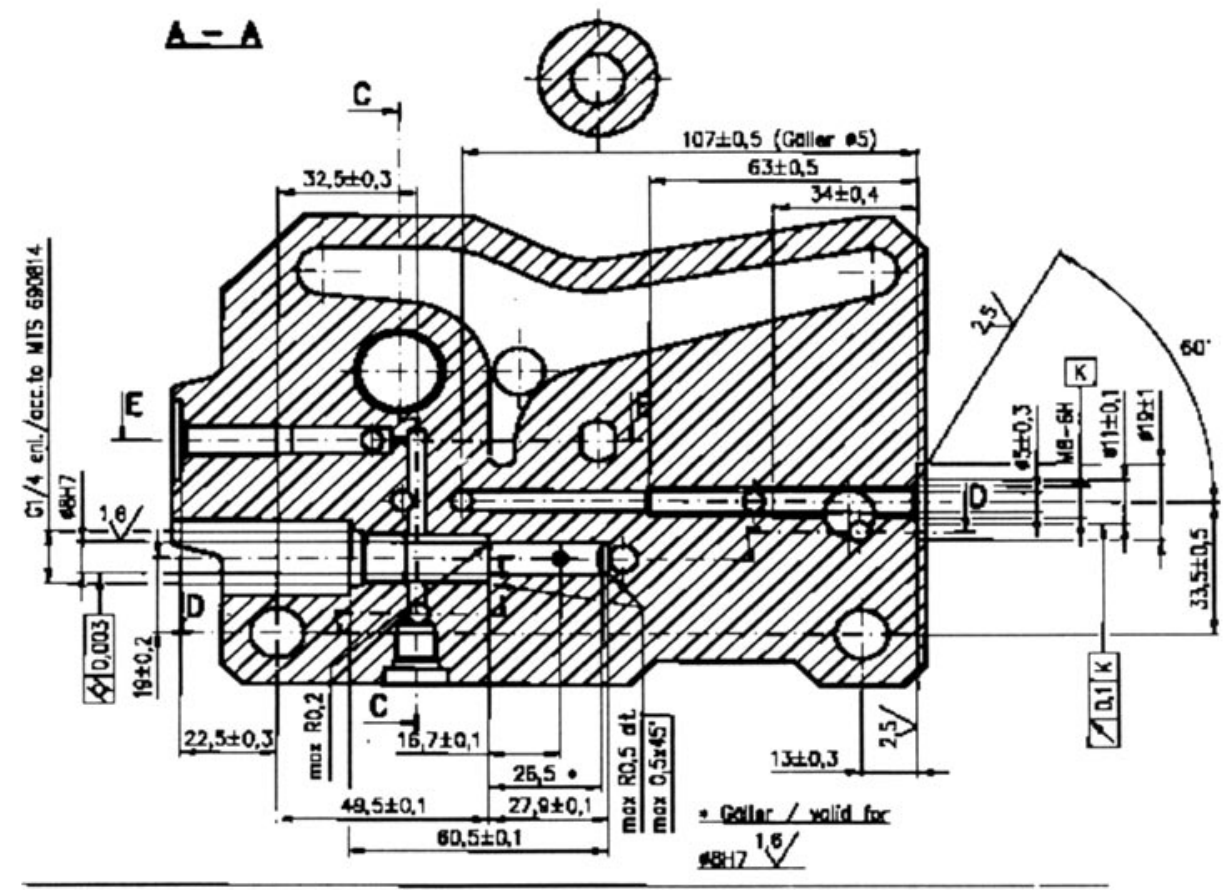

Fig 2. Original raster image of an engineering drawing.

The developed algorithm has been tested in various engineering drawings and the experiments have demonstrated that it yields the best result when the original images of a quite good quality. The thinning operation inevitably introduces some defects, which affects the recognition process. However, the recognition algorithm successfully cope with many of the defects and have a sufficiently high degree of noise immunity. Moreover, using the information obtained at recognition stage makes it possible to keep the percentage of erroneous decisions to a minimum. If the thinned image has too many structural noise, the special algorithms for noise reduction should be applied.

\section{Conclusion}

In this paper, we proposed the two-stage knowledge-based algorithm to recognize crosshatched areas. In the beginning, crosshatched area is detected by using template description and hypothesis advancing, and then a full crosshatched area is extracted. The algorithm has been widely tested experimentally with many ED images and shown good results in crosshatched area recognition. 


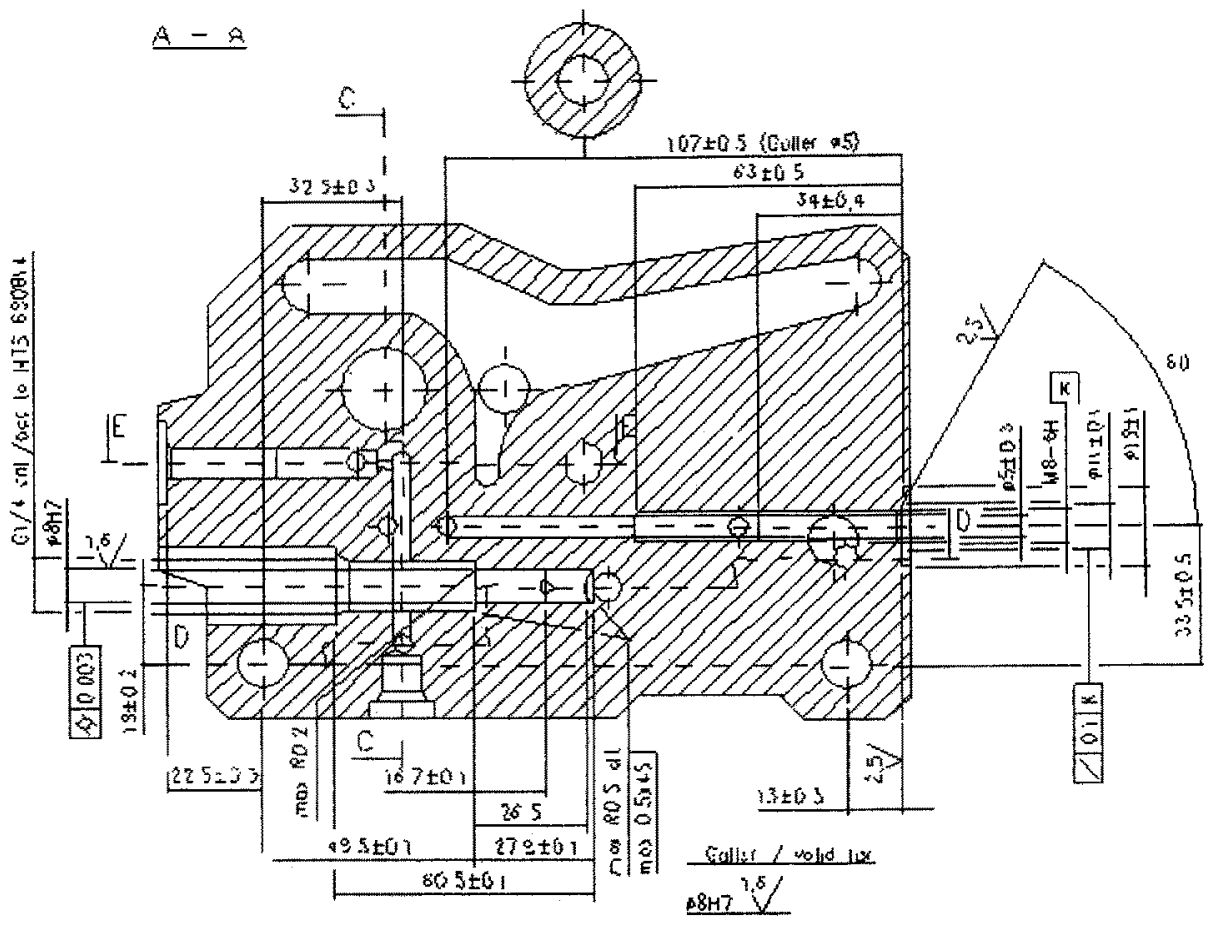

Fig 3. Vectorized image of the drawing shown in Fig.2.
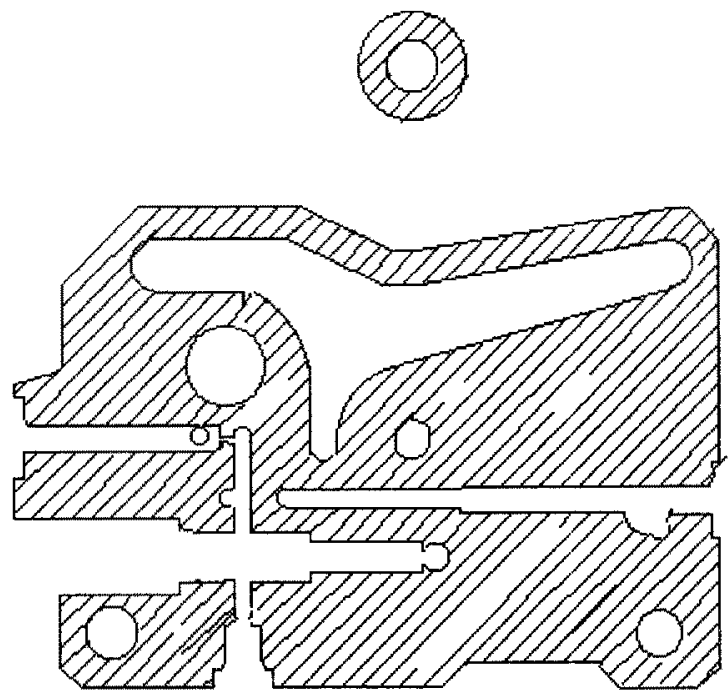

Fig.4. Detected crosshatching areas of the drawing shown in Fig.2. 


\section{References}

1. R.Kasturi, et al., "A system for interpretation of line drawings", IEEE Trans. Pattern Analysis and Machine Intelligence, Vol.12, No.10, pp. 978-992, 1990.

2. V.Nagasamy and N.A.Langrana, "Engineering drawing processing and vectorization system", Computer Vision, Graphics, and Image Processing, Vol.49, pp. 379-397, 1990.

3. M. Ejiri , S. Kakumoto, T.Miyatake, S. Shimads, K. Iwamura "Automatic of engineering drawings and maps", Image Analysis Applications, R. Kasturi and Trivedi, M.M., Eds.,New York: Dekker, 1990.

4. P.Vaxiviere and K.Tombre, "Celesstin: CAD Conversion of Mechanical Drawings", Computer, Vol.25, No.7, pp. 46-54, 1992.

5. S.V.Ablameiko, V.I.Bereishik, O.V.Frantskevich, E.I.Mel'nik, M.I.Khomenko, and N.I.Paramonova, "Interpretation of engineering drawings: techniques and experimental results", Pattern Recognition and Image Analysis, Vol.5, No.3, pp. 380-401, 1995.

6. S.Ablameiko, V.Bereishik et al, System for Automatic Vectorization and Interpretation of Graphic Images, Pattern Recognition and Image Analysis, Vol.3, No.1, 39-52 (1993).

7. S.H.Joseph, T.P.Pridmore, "Knowledge-directed interpretation of mechanical engineering drawings", IEEE Trans. on Pattern Analysis and Machine Intelligence, Vol.14, pp. 928-940, 1992.

8. C.P.Lai and R.Kasturi , "Extraction of objects in engineering drawings", SPIE Vol.2181 Document Recognition, pp. 172-181, 1994.

9. D.Antonie "CIPLAN: A model-based system with original features for understanding French plans", In Proceeding of First International Conference On Document Analysis, IEEE CS, Vol.2,pp. 647-655, 1991.

10. D.Antoine, S.Collin,K.Tombre "Analysis of technical documents: the REDRAW system",Pre-Proc.IAPR Workshop on Structural and Syntactic Pattern Recognition, New Jersey, pp. 192-230,1990.

11. L. Boato et al.. "An interpretation system for land register maps", Computer, Vol.25, N.7, pp25-33, 1992. 